\title{
Constitutive TLR4 signalling in intestinal epithelium reduces tumor load by increasing apoptosis in $\mathrm{APC}^{\mathrm{Min} /+}$ mice.
}

Yinghui $\mathrm{Li}^{1,2}$, Weiling Teo ${ }^{4}$, Meijun Low ${ }^{4}$, Lisa Meijer ${ }^{1}$, Ian Sanderson ${ }^{3}$, Sven Pettersson ${ }^{1,4,5}$, and Gediminas Greicius ${ }^{4}$

Affliations: 1: Department of Microbiology, Tumor and Cell Biology, Karolinska Institute, Theorells vag, S-171 77 Stockholm, Sweden.

2: Singapore Immunology Network (SIgN), Immunos Building, Level 4, Singapore 1386483

3: Digestive Diseases Clinical Academic Unit, Barts and The London, Queen Mary's School of Medicine and Dentistry, London E1 2AT, England, UK.

4: National Cancer Centre, 11 Hospital Drive Singapore 169610.

5: School of Biological Sciences, Nanyang Technological University, 60 Nanyang Drive, Singapore 637551

Corresponding author: Professor Sven Pettersson, Department of Microbiology, Tumor and Cell Biology, Karolinska Institute, Theorells vag, S-171 77 Stockholm, Sweden.

E-mail: Sven.Pettersson@ki.se. Tel: +46 85248 6686. Fax: +46 8331547 


\begin{abstract}
The microbial pattern-recognizing Toll-like receptors (TLRs) play an essential role in shaping the expansion and maturation of the postnatal intestinal epithelium as well as transducing signals from luminal flora to the host. Notably, disruption of this intestinal homeostasis regulated by TLRs affects epithelial cell growth and thus cancer development. To assess the role of TLR4 signalling in epithelial cell proliferation and intestinal homeostasis, we generated a transgenic mouse strain carrying a constitutively active TLR4 (CD4-TLR4) that is linked to an intestinal epithelial cell-specific promoter. CD4-TLR4 transgenic mice had normal intestinal morphology but displayed enhanced Paneth cell functions. Interestingly, crypt-villus organoid cultures derived from CD4-TLR4 mice displayed increased proliferative capacity whilst Cox-2 expression was surprisingly reduced as compared to organoids from non-transgenic, wild-type mice. Introduction of the CD4TLR4 transgene into $\mathrm{APC}^{\mathrm{Min} /+}$ mice, a model of colorectal carcinoma, resulted in a dramatic drop in tumor load in contrast to wild-type $\mathrm{APC}^{\mathrm{Min} /+}$ mice. Furthermore, down-regulation of Cox-2 protein, coincident with elevated IFN $\beta$ expression, resulted in increased levels of apoptosis in CD4-TLR4-APC ${ }^{\mathrm{Min} /+}$ intestinal tumors in vivo. Thus, triggering of microbial signalling via TLR4, at the onset of epithelial cell differentiation, can guide development of the intestinal epithelium by an intricate balance between proliferation and apoptosis.
\end{abstract}




\section{Introduction}

Toll-like receptors (TLRs) are a family of pattern recognition receptors known to regulate host defence against infection, intestinal homeostasis and mucosal innate immune functions. Through their ability to recognize pathogen-associated molecular patterns (PAMPs) found on microbes, TLRs transduce signals from the luminal surface to the intestinal mucosal layer. One major TLR involved in the sensing of gram-negative enteric bacteria and their products such as lipopolysaccharide (LPS) is TLR4. Several studies involving a variety of microbial products and TLR4 agonists have implicated the critical role of TLR4 signalling in the regulation of tumor growth, survival and progression.

In the $\mathrm{APC}^{\mathrm{Min} /+}$ mouse model of spontaneous intestinal tumorigenesis, genetic ablation of MyD88, a downstream signalling adaptor of TLRs, resulted in the reduction of small intestinal tumor frequency and size [1]. This finding thus suggests the potential role of microbial signalling, of which the TLR4 cascade is a major component, in promoting intestinal tumorigenesis, via the MyD88-dependent signalling pathway. Further evidence supporting the positive role of TLR4 in cancer development was demonstrated in a variety of adoptively transferred tumors, where LPS was found to augment tumor growth and metastasis $[2,3,4]$.

While TLR4 signalling has been shown to drive tumorigenesis, recent accumulating evidence has also pointed to an anti-tumor effect of TLR4 activation. Potent anti-cancer effects against adoptively transferred tumors in mice have been illustrated with the administration of LPS [5, 6]. Moreover, the administration of LPS and two other potent TLR4 agonists, OK-432 and Mycobacterium bovis bacillus Calmette-Guérin (BCG), have been shown to be effective in the treatment of various carcinomas such as colorectal, gastric, bladder and lung cancer $[7,8,9,10]$. This negative regulatory role of TLR4 in cancer recapitulates the earlier work by William Coley in the 19th century, where repeated injections of bacterial toxins, later found to be LPS, was effective as an anti-cancer therapeutic [11]. Thus, the role of TLR4 signalling in tumor development is complex and warrants further investigation.

One major mediator of tumor growth and survival is cyclooxygenase 2 (Cox-2). Cyclooxygenases, also known as prostaglandin-endoperoxide synthases (PTGS), are important enzymes that catalyze the synthesis of prostaglandins (PGs) from arachidonic acid [12]. To date, there are two known isoforms of cyclooxgenases, Cox-1 and Cox-2, which are 
differentially expressed and regulated. In contrast to Cox-1, which is expressed ubiquitously in most tissues and generally considered to contribute to cellular homeostasis, Cox-2 expression is rapidly induced by inflammatory mediators, growth factors, oncogenes and endotoxins [12]. Cox-2 is a known critical player of cancer progression in various aspects, including cell proliferation and migration, apoptosis, tumor angiogenesis and metastasis [13, 14]. In the context of colorectal cancer, usage of Cox-2 inhibitors has been efficacious in the treatment of familial adenomatous polyposis (FAP) patients $[15,16]$.

Our recent work as well as studies by other groups has highlighted the crucial role of TLR4 stimulation in promoting tumor cell proliferation and survival in vitro $[17,18,19]$. Thus in this current study, we were interested to understand how TLR4 signalling influences intestinal homeostasis in vivo, in particular, the development of intestinal tumors arising from a genetic predisposition such as the $\mathrm{APC}^{\mathrm{Min} /+}$ mouse model [20]. In our investigation, we developed $\mathrm{APC}^{\mathrm{Min} /+}$ mice expressing constitutively active TLR4 (CD4-TLR4) specifically in the intestinal epithelium. Interestingly, CD4-TLR4 expressing APC ${ }^{\mathrm{Min} /+}$ mice displayed reduced tumor burden in the intestine, and their small intestinal tumors showed increased apoptosis in vivo that correlate with Cox-2 down-regulation. Our findings thus shed light on an unrecognized role of long-term epithelial TLR4 activation in the inhibition of tumorigenesis in the intestine, involving Cox-2 regulation as one of the possible mechanisms. 


\section{Materials and methods}

\section{Animal studies}

CD4-TLR4 transgenic mice were generated using the mCD4-TLR4 fusion gene (a gift from Dr Medzhitov) [21], which comprises a constitutively active human TLR4 cytoplasmic domain fused to the extracellular domain of murine CD4, under the control of intestinal epithelial cell-specific fatty acid binding (I-FABP) promoter in a similar procedure as described previously [22]. Verification of transgenic mice was performed by PCR amplification of genomic DNA using the following primers: 5'-CGCTTCAGTTTGCTG-3', 5'-CCGGGAATAAAGTCTCT-3'. Mice were backcrossed to C57BL/6 mice for over ten generations. To introduce $\mathrm{APC}^{\mathrm{Min} /+}$ mutation, CD4-TLR4-positive females were paired with $\mathrm{APC}^{\mathrm{Min} /+}$ males. Mice of the first generation derived from these crosses were used to assess tumorigenesis. For tumor counting, whole intestine was fixed in PBS-buffered $4 \%$ formalin solution and cut longitudinally. Only clearly defined adenomas exceeding $0.8 \mathrm{~mm}$ were counted as tumors under the dissection microscope. Mice were maintained under specific pathogen free conditions. All animal experiments were performed following Swedish ethical regulations and guidelines of the Institutional Animal Care and Use Committee of SingHealth Office of Research.

\section{Reverse-transcription PCR and quantitative real-time PCR}

Total RNA from mucosal scrapings of different intestinal segments was extracted using the RNeasy mini kit (Qiagen) according to manufacturer's instructions. $2 \mu \mathrm{g}$ total RNA was used for cDNA synthesis using Superscript II (Invitrogen). Transgene expression was analyzed via PCR amplification of $2.5 \mu \mathrm{l}$ cDNA using primers specific for murine CD4 and human TLR4: 5'-GGTTCTGGCAACCTGACTCT-3' and 5'CCCACTGTTCCTTCTGGATT-3'.

Quantitative real-time PCR (qRT-PCR) was performed in triplicates for each sample on a ABI 7500 Real time PCR System (Applied Biosystems) using Fast SYBR Green PCR Mastermix (Applied Biosystems). Mouse primer sequences for the various genes described in the study are presented in Supplementary table 1. Q-PCR data are presented as fold change of relative gene expression using $\beta$-actin as endogenous control. 


\section{Immunostaining}

Colonic tissues from CD4-TLR4 and wild-type (WT) mice were freshly embedded in OCT (Tissue-Tek 4583), snap frozen and sectioned on a cryostat. Sections were blocked in $10 \%$ mouse serum for $30 \mathrm{~min}$ at room temperature before incubating with CD4 antibody (BD Pharmingen, 550278) at 1:50 dilution for $1 \mathrm{~h}$ at room temperature. The slides were then washed in PBS and incubated with Alexa Fluor 488 goat anti-rat IgG (Invitrogen, A11006). 4',6-diamidino-2-phenylindole (Dapi) was used for nuclear staining. For staining with Ki-67 antibody (Leica, NCL-Ki-67-MM1), formalin-fixed intestinal tissues from CD4-TLR4 and wild-type (WT) mice were used. Sections were deparaffinised in Bond Dewax solution (Leica) before performing antigen retrieval using Bond Epitope Retrieval Solution (Leica).

\section{Crypt isolation, cell dissociation and culture}

S1-3 small intestinal segments (refer to Figure S1B for classification) from CD4TLR4 and WT mice were collected for crypt isolation and culture as described previously [23]. 6000 crypts per well on 48-well plates were cultured as described previously [23]. For culture experiments, four independent experiments were performed. For each culture/sorting experiment, crypts were pooled from 2-5 intestines. At day 6 from initial plating of crypts, total RNA was isolated from cultures using Trizol LS reagent (Invitrogen) according to manufacturer's recommendations.

For sorting of Paneth cells, crypts were dissociated with $0.5 \mathrm{X}$ trypsin for $40 \mathrm{~min}$ at $37^{\circ} \mathrm{C}$, passed through a $40 \mu \mathrm{m}$ cell strainer (BD) and washed with PBS/2\% FBS. Cells were incubated with anti-CD24 antibody (BD Pharmingen, 553260) for $50 \mathrm{~min}$ at $4^{\circ} \mathrm{C}$, washed with PBS/2\% FBS and then incubated with Alexa-fluor goat anti-rat IgG for $30 \mathrm{~min}$ at $4^{\circ} \mathrm{C}$. Viable epithelial single cells were gated by forward scatter, side scatter and pulse-width parameter, and negative staining for dapi. Cells were collected in cold PBS containing 20\% FBS, and total RNA was extracted using PicoPure RNA isolation kit (Arcturus) according to manufacturer's instructions.

\section{In vivo apoptosis assay}

To detect apoptosis in vivo, CD4-TLR4-APC ${ }^{\mathrm{Min} /+}$ and $\mathrm{APC}^{\mathrm{Min} /+}$ mice were injected intravenously with FAM-FLIVO (Immunochemistry, 981) via tail vein $1 \mathrm{~h}$ prior to sacrifice and harvesting of intestinal tissues. Whole intestines were fixed in PBS-buffered $4 \%$ formalin solution and cut longitudinally. Tissues sections of tumors and normal epithelia were 
subsequently made and apoptotic cells were visualized under a fluorescence microscope at an excitation wavelength of $488 \mathrm{~nm}$. At least 10 different small intestinal tumors and 60 nonoverlapping microscopic fields of view under a $20 \mathrm{X}$ objective from each group were analyzed and counted for apoptotic cells.

\section{Statistical analysis}

GraphPad Prism 5 was used for all statistical tests performed in this study. 


\section{Results}

\section{Constitutive epithelial TLR4 signalling suppresses APC ${ }^{\mathrm{Min} /+}$ intestinal tumorigenesis}

In our recent study of $\mathrm{APC}^{\mathrm{Min} /+}$ mice, we established microbial signalling as a major mediator of intestinal tumorigenesis [17]. This finding complements previous work from Medzhitov's group, whereby deletion of MyD88 resulted in a dramatic reduction of tumor burden in $\mathrm{APC}^{\mathrm{Min} /+}$ mice [1]. Moreover, we further identified TLR4, a key component of microbial signalling, as a regulator of tumor cell proliferation [17]. We thus sought to further dissect its role in intestinal tumorigenesis in vivo. To address this, we generated mice transgenic for CD4-TLR4, which comprised of murine CD4 ectodomain fused to constitutively active domains of human TLR4 [21]. To avoid interference with the intestinal stem cell compartment, the CD4-TLR4 transgene was linked to the intestinal fatty acid binding protein (I-FABP) promoter, which is only expressed in mature epithelial lineages of the intestine [24, 25]. Analysis of various intestinal segments by RT-PCR and Q-PCR showed that CD4-TLR4 transgenic mice express the transgene throughout the gut as early as 4 weeks of age (Figure S1A), with highest expression levels occurring predominantly in the proximal small intestine and colon (Figure S1B). Immunostaining of intestinal sections with anti-mouse CD4 antibody verified epithelial localization of the fusion protein in transgenic but not in control mice (Figure S1C). The CD4-TLR4 mice had normal lifespan and did not exhibit abnormalities in intestinal morphology or abnormal influx of inflammatory cells (Figure S1D). Thus constitutive TLR4 signalling in intestinal epithelia does not induce spontaneous inflammation nor affect intestinal function and morphology.

We next crossed CD4-TLR4 mice with $\mathrm{APC}^{\mathrm{Min} /+}$ mice, and analyzed for tumor load in the intestines of these mice. Unexpectedly, we found that $\mathrm{APC}^{\mathrm{Min} /+}$ mice expressing constitutively active TLR4 developed significantly fewer tumors in small intestine than $\mathrm{APC}^{\mathrm{Min} /+}$ mice at both $16-$ and 28 - weeks of age $(\mathrm{P}=5.432 \mathrm{e}-05$ and $\mathrm{P}=0.0023$ respectively, Mann-Whitney) (Figure 1A and S2A). Mean tumor size was also significantly reduced in these mice as compared to age-matched $\mathrm{APC}^{\mathrm{Min} /+}$ mice at 16 weeks of age $(\mathrm{P}=1.881 \mathrm{e}-12$, Mann-Whitney test) (Figure 1B).

Transgene expression enhances Paneth cell functions leading to increased epithelial growth in long-term ex vivo cultures.

As we were surprised by the dramatic reduction of tumor load in CD4-TLR4$\mathrm{APC}^{\mathrm{Min} /+}$ mice, we examined whether long-term, in vivo, epithelial TLR4 stimulation 
influenced intestinal epithelial proliferation. Immunohistochemical analysis of Ki-67 revealed increased nuclear staining in intestinal epithelial cells residing above crypt bases of CD4TLR4 mice as compared to WT mice (Figure 2A), indicating a higher proliferative status of the intestinal epithelium during persistent TLR4 activation.

We then sought to investigate how constitutive epithelial TLR4 signalling can influence the stem cell compartment, thus impacting on epithelial proliferation. As Paneth cells have been demonstrated to be critical for the maintenance and ability of Lgr5+ intestinal stem cells to form self-organizing crypt-villus organoids through producing essential niche signals [26], we checked our CD4-TLR4 mice for Paneth cell functions. Periodic acid-Schiff (PAS) staining of intestinal segments of WT and CD4-TLR4 mice showed more abundant staining of Paneth cell-like cells at colonic crypt bases as well as goblet cells at colonic crypts of CD4-TLR4 mice as compared to age-matched, WT counterparts (Figure S3, upper panel). In contrast, we did not detect visible differences in Paneth cells and goblet cells amounts in the small intestines of CD4-TLR4 and WT mice (Figure S3, lower panel).

Consistent with increased Paneth cell levels, CD4-TLR4 mice expressed elevated mRNA expression of antimicrobial peptides, cryptdin- 1 and lysozyme, in colonic mucosal scrapings as compared to WT mice and this was correlated with high expression levels of the transgene ( $>8$-fold induction compared to lowest transgene expressing sample) $(\mathrm{P}<0.01$, Mann-Whitney) (Figure 2B). Similar observations were found for relative expression levels of Wnt1 and Wnt3, which are known to be produced by Paneth cells [27] ( $\mathrm{P}<0.01$, MannWhitney) (Figure 2B). The expression levels of proliferating cell nuclear antigen (PCNA) between WT and CD4-TLR4 colonic mucosal scrapings were however not significantly different (Figure 2B).

To more precisely examine whether increased Paneth cell activity and persistent TLR4 stimulation had a proliferative impact on the intestinal epithelium, we purified intestinal crypts from CD4-TLR4 and WT mice and cultured them ex vivo for 5 days using an established matrigel-based culture system [26]. As regions S1-3 of the small intestines of CD4-TLR4 mice were found to express the highest levels of transgene (Figure S1B), we used the S1-3 intestinal segments from both CD4-TLR4 and WT mice for our crypt isolation studies. We found that while the ability to form viable crypt-villus organoids a day after initial plating did not differ between CD4-TLR4 and WT intestinal crypts, CD4-TLR4 organoids appeared to be larger and more viable than WT organoids after 5 days of culture, forming new crypts from budding sites (Figure 3A). Moreover, Ki-67 staining showed denser 
nuclear staining in the villus regions of CD4-TLR4 organoids as compared to WT organoids (Figure 3B).

We further performed real-time PCR expression analyses of our crypt-villus organoid cultures after 5 days and observed that CD4-TLR4 organoids maintained elevated levels of Paneth cell markers - cryptdin-1, lysozyme and Wnt3 in comparison to WT organoids (Figure 3C), similar to our earlier findings from mucosa scrapings (Figure 2B). Interestingly, a strong induction of Lgr5 and PCNA was observed in CD4-TLR4 organoids as compared to WT organoids (Figure 3C), which is consistent with the current notion that increased Paneth cell levels promote the maintenance and development of intestinal stem cells, as well as epithelial cell growth.

We then asked whether increased Paneth cell activity in CD4-TLR4 organoids was due to constitutive TLR4 stimulation in Paneth cells by performing FACS sorting of CD24+ Paneth cells from purified intestinal crypts of CD4-TLR4 mice and probing for transgene expression in sorted Paneth cells. As shown in Figure S4, sorted CD24 $4^{\text {high }}$ cells (P8 fraction) were found to express elevated levels of Paneth cell markers - cryptdin-1, lysozyme and defcr-rs-10 as compared to CD24 $4^{\text {low }}$ cells (P5 and P6 fractions). Notably, sorted CD24 $4^{\text {high }}$ Paneth cells expressed higher levels of transgene than CD24 ${ }^{\text {low }}$ cells (Figure S4B). Taken together, our findings demonstrate that constitutive TLR4 signalling in the intestinal epithelium drives epithelial cell proliferation through enhanced Paneth cell functions in vivo. Moreover, CD4-TLR4 organoids expressed higher levels of chromogranin A (CHGA) and mucin 2 (Muc2) which are known markers of enteroendocrine and goblet cells respectively (Figure 3C), demonstrating that persistent epithelial TLR4 activation leads to enhanced functions of all secretory lineages in the intestine.

\section{Reduced Cox-2 expression correlates with increased apoptosis levels of CD4-TLR4 expressing intestinal tumors}

While we observed that increased proliferation in our ex vivo organoid cultures is correlated with active TLR4 signalling, we were surprised to find reduced Cox-2 levels in CD4-TLR4 organoids after 5 days of culture (Figure 3C). As Cox-2 is an important player of tumorigenesis [28, 29, 30], we investigated levels of transgene and Cox-2 in small intestinal tumors of CD4-TLR4-APC ${ }^{\mathrm{Min} /+}$ mice. Subsequent real-time pcr analyses revealed an inverse correlation between transgene expression and Cox-2 levels in CD4-TLR4-APC ${ }^{\mathrm{Min} /+}$ tumors (Figure 4A). Notably, Cox-2 expression was significantly reduced in CD4-TLR4-APC ${ }^{\mathrm{Min} /+}$ tumors as compared to tumors from age-matched $\mathrm{APC}^{\mathrm{Min} /+}$ mice (Figure 4B; $\mathrm{P}<0.01$, Mann- 
Whitney), consistent with lower protein levels of Cox-2 in tumor lysates (Figure S5). This down-regulation of Cox-2 expression correlated with an increased expression of proapoptotic markers, Bad and p21 (Figure 4C). Interestingly, IFN $\beta 1$, a type I interferon (IFN) found to contribute positively to antitumoral immune responses in TLR4-activated tumors [31], was markedly up-regulated (10-fold increase) in CD4-TLR4-APC ${ }^{\mathrm{Min} /+}$ tumors as compared to $\mathrm{APC}^{\mathrm{Min} /+}$ tumors (Figure 4B; $\mathrm{P}<0.001$, MannWhitney). Subsequent analysis of these tumors for markers of proliferation and various secretory cell lineages showed that despite an increased expression of Wnt3 in CD4-TLR4-APC ${ }^{\mathrm{Min} /+}$ tumors, expression levels of PCNA and Paneth cell markers, cryptdin-1 and lysozyme, were not different from that of $\mathrm{APC}^{\mathrm{Min} /+}$ tumors (Figure 4D). Meanwhile, increased Muc2 expression was observed in CD4TLR4-APC ${ }^{\mathrm{Min} /+}$ tumors while CHGA levels remained unchanged (Figure 4D).

As Cox-2 is known to be an important mediator of tumor cell survival through the inhibition of apoptosis [13, 14], we probed whether Cox-2 down-regulation in CD4-TLR4 expressing mice increased levels of apoptosis in normal and transformed epithelial cells in vivo. We injected WT and CD4-TLR4 mice, as well as $\mathrm{APC}^{\mathrm{Min} /+}$ and transgene-expressing $\mathrm{APC}^{\mathrm{Min} /+}$ mice with FAM-FLIVO, a green fluorescent probe (carboxyfluorescein) that binds irreversibly to active caspases [32], and analyzed for levels of apoptotic cells in normal mucosa and tumor tissues of the small intestine. We found that while small intestinal tumors did not appear histologically different between WT and CD4-TLR4-expressing APC ${ }^{\mathrm{Min} /+}$ mice (Figure 5A), levels of apoptotic cells in CD4-TLR4-APC ${ }^{\mathrm{Min} /+}$ tumors were markedly higher than age-matched, non-transgenic $\mathrm{APC}^{\mathrm{Min} /+}$ mice (Figure 5B and 5C; $\mathrm{P}<0.001$, MannWhitney). In contrast, no significant differences in levels of apoptotic cells were observed in the normal mucosa of CD4-TLR4 mice as compared to age-matched WT mice (Figure S6). Collectively, these findings thus demonstrate an elevated level of apoptosis in CD4-TLR4expressing intestinal tumor cells in vivo, that correlate with Cox-2 down-regulation.

\section{Discussion}

The interaction of commensal microflora with the host via TLR signalling is critical not only for host defense against pathogens, but also for gut epithelial homeostasis such as the maintenance of a healthy epithelial barrier and regulation of epithelial cell proliferation. Thus in our study, we employed constitutively active TLR4 as an experimental approach to simulate host-microbe signalling in vivo and in turn understand its role in regulating epithelial homeostasis and tumorigenesis. Through introduction of epithelial CD4-TLR4 in the 
$\mathrm{APC}^{\mathrm{Min} /+}$ mouse model of intestinal tumorigenesis, our findings reveal the intricate balance between epithelial proliferation and apoptosis that is regulated by microbial signalling. This fine equilibrium of proliferation versus programmed cell death modulates aberrant epithelial cell growth and hence the development of intestinal tumors.

In our study, we found that persistent epithelial TLR4 activation impacts on Paneth cell functions, as well as all other secretory cell lineages in the intestine, thus regulating epithelial cell growth and intestinal homeostasis. While constitutive TLR4 signalling results in an increased proliferative status of the intestinal epithelium, a higher extent of apoptosis also ensues in a genetically predisposed environment, possibly through down-regulation of critical survival factor, Cox-2. Thus as an indirect consequence of constitutive TLR4 signalling, the turnover rate of transformed intestinal epithelial cells is enhanced and this disrupted balance of proliferation versus apoptosis tips over towards the latter, leading to the suppression of $\mathrm{APC}^{\mathrm{Min} /+}$-driven spontaneous tumorigenesis.

Using the $\mathrm{APC}^{\mathrm{Min} /+}$ mouse model of spontaneous intestinal tumorigenesis, we found that the expression of constitutively active TLR4 in intestinal epithelium results in the suppression of intestinal tumor outgrowth (Figure 1 and S2A). Although mice transgenic for CD4-TLR4 in the intestinal epithelium i.e. villin-TLR4 mice have been studied previously in a colitis-associated neoplasia model [33], we used a different promoter, the intestinal fatty acid binding protein (I-FABP) promoter, which is only activated when differentiating enterocytes leave the crypts and migrate to the base of small intestinal villi or surface of colonic epithelia $[24,25]$. As such, in contrast to the villin promoter, I-FABP promoter circumvents interference of the active transgene with stem cell development and early differentiation of the four epithelial lineages at intestinal crypt bases. Thus, we verified transgene expression and their epithelial localization in the intestine of these mice (Figure S2A-C).

While we were surprised to find a remarkable reduction of tumor burden in CD4TLR4-expressing $\mathrm{APC}^{\mathrm{Min} /+}$ mice (Figure 1 and S3A), our data from intestinal mucosal tissues and ex vivo organoid cultures support current, cumulative evidence for the major contribution of TLR4 signalling in epithelial cell proliferation $[3,4,17,18]$. Notably, we found that constitutively active epithelial TLR4 resulted in increased proliferating cells (Figure 2A) correlating with increased Paneth cells (Figure S3) and elevated expression of Paneth cell markers (cryptdin-1 and lysozyme) and Wnts in the intestine (Figure 2B). This increased proliferative status was further recapitulated in our ex vivo organoid cultures (Figure 3). Meanwhile, this increased proliferative capacity did not result in aberrant crypt outgrowth in 
the transgenic mice (Figure S1D), suggesting that additional regulatory signals, such as enhanced functions of all secretory cell lineages (Figure 3C), are in place to prevent aberrant epithelial growth and maintain normal intestinal homeostasis.

Although a recent study has shown that Lgr5+ intestinal stem cells can function normally in the absence of Paneth cells [34], our studies support accumulating evidence for the major contribution of Paneth cells in intestinal stem cell survival and self renewal functions [26, 35]. Moreover, the identification of transgene expression in FACS-sorted Paneth cells from purified CD4-TLR4 expressing crypts, coupled with our ex vivo organoid cultures, suggest the role of active TLR4 signalling in stimulating Paneth cell activity and thus intestinal epithelial proliferation. However, we do not rule out the possibility that Paneth cells are not the sole producers of essential niche signals for Lrg5 stem cells.

While we observed proliferative effects associated with active TLR4 stimulation, one intriguing finding arising from our ex vivo organoids culture work was the regulation of TLR4 on Cox-2 expression. Although current cumulative evidence support the notion that TLR4 induces Cox-2 expression in both intestinal epithelial cells and lamina propria stromal cells as an important homeostatic response triggered during inflammatory stimulation [36, 37], our study suggests that chronic stimulation of TLR4 in intestinal epithelial cells may lead to the downregulation of Cox-2 instead (Figure 3C).

This interesting phenomenon provides a plausible mechanism underlying the reduced tumor load observed in our CD4-TLR4-APC ${ }^{\mathrm{Min} /+}$ mice (Figure 1 and S2A). We found significantly reduced levels of Cox-2 expression in intestinal tumors from CD4-TLR4$\mathrm{APC}^{\mathrm{Min} /+}$ mice as compared to tumors from $\mathrm{APC}^{\mathrm{Min} /+}$ mice, which correlated with high transgene expression (Figure 4A-B and S5). This finding thus supports the role of Cox-2 inhibitors in the amelioration of intestinal tumors in $\mathrm{APC}^{\mathrm{Min} /+}$ mice as well as in familial adenomatous polyposis (FAP) patients $[15,16]$. Moreover, other epithelial models of Cox-2 overexpression using both cancer cell lines and transgenic mice have demonstrated the role of Cox-2 in promoting tumor development $[38,39]$.

Accumulating evidence has pointed to the significant contribution of Cox-2 in tumor promotion by imparting resistance to apoptosis $[13,14]$. One study has also reported that mice expressing the CD4-TLR4 construct under a different epithelial promoter developed significantly more azoxymethane (AOM)-induced tumors when mucosal Cox-2 levels were significantly up-regulated by repeated cycles of DSS-induced colitis [21]. In contrast, our in vivo studies of CD4-TLR4-APC ${ }^{\mathrm{Min} /+}$ mice support the role of Cox-2 in the regulation of apoptosis when mere TLR4 activation in epithelial cells is not supported by concomitant 
inflammation. Notably, the down-regulation of Cox-2 in CD4-TLR4 expressing intestinal tumors was closely associated with an increased expression of apoptotic markers -Bad and p21 (Figure 4C). This increased apoptotic potential was further highlighted in vivo by a marked increase in apoptotic cells per tumor area in the small intestinal tumors of CD4TLR4-APC $^{\mathrm{Min} /+}$ mice (Figure 5B-C), thereby suggesting that Cox-2 inhibition promotes the apoptosis of intestinal epithelial cells leading to decreased survival of CD4-TLR4-APC ${ }^{\mathrm{Min} /+}$ tumors.

Interestingly, the expression of IFN $\beta 1$, a key host defense cytokine against pathogens including microbes and tumor cells, is also upregulated in these CD4-TLR4 tumors (Figure 4B). While IFN $\beta$ has been recently identified to be produced by TLR4-activated tumor cells as a critical mediator of the antitumoral immune response [31], the synergistic effect of Cox2 inhibitor and IFN $\beta$ in the induction of apoptosis has been demonstrated previously to be efficacious in tumor cell lines and xenotransplanted tumors [40]. Moreover, IFN $\beta$ has been shown to inhibit Cox-2 expression at the transcriptional level in intestinal epithelial cells [41]. These findings thus provide a possible mechanism for the increased levels of apoptotic cells detected in CD4-TLR4 tumors, resulting in the overall suppression of intestinal tumorigenesis. This reduced tumor burden of CD4-TLR4-APC ${ }^{\mathrm{Min} /+}$ mice was in spite of enhanced mucosal expression of growth factors (Wnts), and Paneth cell functions which supports epithelial cell proliferation. These observations thus put forth an interesting notion that chronic TLR4 activation counteracts growth signals in the intestinal epithelium through triggering apoptosis, which in turn influences tumor initiation and survival, and this is likely to involve Cox-2 as one of the regulatory mechanisms. 


\section{Acknowledgements}

Our research work was funded by a grant from Singapore Immunology Network (SIgN) SigN 10-038. We would like to thank Dr Robert Wallin for his great expertise and assistance in using the deconvolution microscope for anti-CD4 fluorescence imaging. We thank Velmurugesan Arulampalam for valuable discussions about our study. We would also like to thank Arlaine Anne Bautista Amoyo, Jieshun Wong and Annika Samuelsson for their technical support with animal care and handling. 


\section{Figure legends}

Figure 1. Constitutive TLR4 signalling in intestinal epithelium inhibits $\mathrm{APC}^{\mathrm{Min} /{ }^{-}}$-driven intestinal tumorigenesis. (A) CD4-TLR4-APC ${ }^{\mathrm{Min} /+}$ mice $(\mathrm{n}=11)$ have reduced tumor load in both colon (right panel, asterisk represent $\mathrm{P}<0.05$, Fisher's test) and small intestine (left panel, asterisk represent $\mathrm{P}<0.05$, Mann-Whitney) at 16 weeks of age as compared to agematched $\mathrm{APC}^{\mathrm{Min} /+}$ mice $(\mathrm{n}=12)$. (B) Distribution of tumor sizes and counts in CD4-TLR4$\mathrm{APC}^{\mathrm{Min} /+}$ mice and $\mathrm{APC}^{\mathrm{Min} /+}$ mice at 16 weeks of age (asterisk represent $\mathrm{P}<0.01$, MannWhitney, $\mathrm{n}=105$ and $\mathrm{n}=435$ for small intestinal tumors derived from CD4-TLR4-APC ${ }^{\mathrm{Min} /+}$ and $\mathrm{APC}^{\mathrm{Min} /+}$ mice respectively.

Figure 2. Transgene expression in intestinal epithelium leads to increased epithelial cell proliferation and enhanced Paneth cell functions. (A) Ki-67 staining of colon and small intestine of age-matched CD4-TLR4 and WT mice. (B) Relative expression levels of Paneth cell markers (Wnt1, Wnt3, cryptdin-1 and lysozyme) and PCNA in colonic mucosal scrapings of CD4-TLR4 $(n=18)$ and WT mice $(n=8)$ were analyzed by qRT-PCR. CD4-TLR4 samples were further categorized according to levels of transgene expression: CD4-TLR4 low (relative fold change 1-8) (n=9) and CD4-TLR4 high (relative fold change >8) (n=9). All asterisks represent $\mathrm{P}<0.01$, Mann-Whitney test.

Figure 3. Transgene expression in intestinal epithelium results in enhanced functions of all secretory cell lineages and promotes epithelial cell proliferation ex vivo. (A) Representative pictures of ex vivo intestinal crypt organoid cultures of CD4-TLR4 (lower panels) and WT (upper panels) mice following one and five days of plating as indicated. (B) CD4-TLR4 organoids (right column) display more abundant Ki-67 nuclear staining than WT (left column) organoids. (C) CD4-TLR4 organoids express elevated levels of markers for Paneth cells (Wnt3, cryptdin-1 and lysozyme), proliferation (PCNA), intestinal stem cells (Lrg5), enteroendocrine cells (CHGA) and goblet cells (Muc2) while Cox-2 expression in downregulated in comparison to WT organoids. Graphs represent datasets from one out of 3 independent experiments.

Figure 4. CD4-TLR4-expressing $\mathrm{APC}^{\mathrm{Min} /+}$ tumors have reduced Cox-2 expression which correlates with increased levels of apoptotic markers. (A) Inverse correlation between transgene expression and Cox-2 levels in intestinal tumors from CD4-TLR4-APC ${ }^{\mathrm{Min} /+}$ mice $(n=8)$. (B) Transgene-expressing intestinal tumors from CD4-TLR4-APC ${ }^{\text {Min/+ }}$ mice $(n=8)$ 
have reduced Cox-2 levels and increased IFN $\beta 1$ expression than tumors from $\mathrm{APC}^{\mathrm{Min} /+}$ mice $(\mathrm{n}=8)$. Asterisks represent $\mathrm{P}<0.01$ and $\mathrm{P}<0.001$ respectively, Mann-Whitney test. (C) Intestinal tumors from CD4-TLR4-APC ${ }^{\mathrm{Min} /+}$ mice expressing low Cox-2 levels display increased expression of apoptotic markers, Bad and p21, as compared to tumors from $\mathrm{APC}^{\mathrm{Min} /+}$ mice. (D) Relative expression levels of proliferation- and various secretory cell lineage markers in small intestinal tumors from $\mathrm{APC}^{\mathrm{Min} /+}(\mathrm{n}=8)$ and CD4-TLR4-APC $\mathrm{Min/+}$ mice $(\mathrm{n}=8)$. Asterisks represent $\mathrm{P}<0.01$ and $\mathrm{P}<0.1$ for $\mathrm{Wnt} 3$ and Muc2 respectively, MannWhitney test.

Figure 5. CD4-TLR4-APC $\mathrm{Min/+}$ mice display increased apoptosis in normal and transformed epithelial cells. (A) H\&E staining of small intestinal tumors from CD4-TLR4-APC ${ }^{\mathrm{Min} /+}$ and $\mathrm{APC}^{\mathrm{Min} /+}$ mice. (B) Representative images of in vivo labelled apoptotic cells (green) in small intestinal tumors of $\mathrm{APC}^{\mathrm{Min} /+}$ and CD4-TLR4-APC ${ }^{\mathrm{Min} /+}$ mice. (C) Counts of apoptotic epithelial cells per small intestinal tumor area of CD4-TLR4-APC ${ }^{\mathrm{Min} /+}$ versus $\mathrm{APC}^{\mathrm{Min} /+}$ mice. Asterisk represent $\mathrm{P}<0.001$, Mann Whitney test. 


\section{References}

1 Rakoff-Nahoum S, Medzhitov R. Regulation of Spontaneous Intestinal Tumorigenesis Through the Adaptor Protein MyD88. Science 2007;317:124-7.

2 Luo J-L, Maeda S, Hsu L-C, et al. Inhibition of NF-k B in cancer cells converts inflammationinduced tumor growth mediated by TNF $\alpha$ to TRAIL-mediated tumor regression. Cancer Cell 2004;6:297-305.

3 Pidgeon GP, Harmey JH, Kay E, et al. The role of endotoxin/lipopolysaccharide in surgically induced tumour growth in a murine model of metastatic disease. Br J Cancer 1999;81:1311-7.

4 Harmey JH, Bucana CD, Lu W, et al. Lipopolysaccharide-induced metastatic growth is associated with increased angiogenesis, vascular permeability and tumor cell invasion. International Journal of Cancer 2002;101:415-22.

5 Chicoine $\mathrm{M}$, Zahner $\mathrm{M}$, Won $\mathrm{E}$, et al. The in vivo antitumoral effects of lipopolysaccharide against glioblastoma multiforme are mediated in part by Toll-like receptor 4. Neurosurgery 2007;60:372-80.

6 Andreani V, Gatti G, Simonella L, et al. Activation of Toll-like Receptor 4 on Tumor Cells In vitro Inhibits Subsequent Tumor Growth In vivo. Cancer Research 2007;67:10519-27.

7 Maehara $Y$, Okuyama T, Kakeji $Y$, et al. Postoperative immunochemotherapy including streptococcal lysate OK-432 is effective for patients with gastric cancer and serosal invasion. The American Journal of Surgery 1994;168:36-40.

8 Sato $\mathrm{M}$, Harada $\mathrm{K}$, Yoshida $\mathrm{H}$, et al. Therapy for oral squamous cell carcinoma by tegafur and streptococcal agent OK-432 in combination with radiotherapy: association of the therapeutic effect with differentiation and apoptosis in the cancer cells. Apoptosis 1997;2:227-38.

9 Otto F, Schmid P, Mackensen A, et al. Phase II trial of intravenous endotoxin in patients with colorectal and non-small cell lung cancer. European Journal of Cancer 1996;32A:1712-8.

10 Razack AHA. Bacillus Calmette-Guérin and Bladder Cancer. Asian Journal of Surgery 2007;30:302-9.

11 Garay RP, Viens P, Bauer J, et al. Cancer relapse under chemotherapy: Why TLR2/4 receptor agonists can help. European Journal of Pharmacology 2007;563:1-17.

12 Smith WL, DeWitt DL, Garavito RM. CYCLOOXYGENASES: Structural, Cellular, and Molecular Biology. Annual Review of Biochemistry 2000;69:145-82.

13 Trifan OC, Hla T. Cyclooxygenase-2 modulates cellular growth and promotes tumorigenesis. Journal of Cellular and Molecular Medicine 2003;7:207-22.

14 Wang D, DuBois RN. Prostaglandins and cancer. Gut 2006;55:115-22.

15 Steinbach G, Lynch PM, Phillips RKS, et al. The Effect of Celecoxib, a Cyclooxygenase-2 Inhibitor, in Familial Adenomatous Polyposis. New England Journal of Medicine 2000;342:1946-52.

16 Swamy MV, Patlolla JMR, Steele VE, et al. Chemoprevention of Familial Adenomatous Polyposis by Low Doses of Atorvastatin and Celecoxib Given Individually and in Combination to APCMin Mice. Cancer Research 2006;66:7370-7.

17 Li Y, Kundu P, Seow SW, et al. Gut Microbiota accelerate Tumor Growth via c-Jun and STAT3 Phosphorylation in APCMin/+ Mice. . Carcinogenesis 2012.

18 Yokota S-i, Okabayashi T, Rehli M, et al. Helicobacter pylori Lipopolysaccharides Upregulate Toll-Like Receptor 4 Expression and Proliferation of Gastric Epithelial Cells via the MEK1/2-ERK1/2 Mitogen-Activated Protein Kinase Pathway. Infection and Immunity 2010;78:468-76.

19 Bohnhorst J, Rasmussen T, Moen SH, et al. Toll-like receptors mediate proliferation and survival of multiple myeloma cells. Leukemia 2006;20:1138-44.

20 Moser A, Pitot $\mathrm{H}$, Dove W. A dominant mutation that predisposes to multiple intestinal neoplasia in the mouse. Science 1990;247:322-4.

21 Medzhitov R, Preston-Hurlburt P, Janeway CA. A human homologue of the Drosophila Toll protein signals activation of adaptive immunity. Nature 1997;388:394-7. 
22 Arulampalam V, Grant PA, Samuelsson A, et al. Lipopolysaccharide-dependent transactivation of the temporally regulated immunoglobulin heavy chain $3^{\prime}$ enhancer. European Journal of Immunology 1994;24:1671-7.

23 Sato T, Vries RG, Snippert HJ, et al. Single Lgr5 stem cells build cryptÂ-villus structures in vitro without a mesenchymal niche. Nature 2009;459:262-5.

24 Cohn SM, Simon TC, Roth KA, et al. Use of transgenic mice to map cis-acting elements in the intestinal fatty acid binding protein gene (Fabpi) that control its cell lineage-specific and regional patterns of expression along the duodenal-colonic and crypt-villus axes of the gut epithelium. The Journal of Cell Biology 1992;119:27-44.

25 Sweetser D, Hauft S, Hoppe P, et al. Transgenic mice containing intestinal fatty acid-binding protein-human growth hormone fusion genes exhibit correct regional and cell-specific expression of the reporter gene in their small intestine. Proc Natl Acad Sci U S A 1988;85:9611-5.

26 Sato T, van Es JH, Snippert HJ, et al. Paneth cells constitute the niche for Lgr5 stem cells in intestinal crypts. Nature 2011;469:415-8.

27 Gregorieff A, Pinto D, Begthel H, et al. Expression Pattern of Wnt Signaling Components in the Adult Intestine. Gastroenterology 2005;129:626-38.

28 Kargman SL, O'Neill GP, Vickers PJ, et al. Expression of Prostaglandin G/H Synthase-1 and -2 Protein in Human Colon Cancer. Cancer Research 1995;55:2556-9.

29 Kutchera W, Jones DA, Matsunami N, et al. Prostaglandin H synthase 2 is expressed abnormally in human colon cancer: evidence for a transcriptional effect. Proceedings of the National Academy of Sciences 1996;93:4816-20.

30 Sheng $\mathrm{H}$, Shao J, Kirkland SC, et al. Inhibition of human colon cancer cell growth by selective inhibition of cyclooxygenase-2. The Journal of Clinical Investigation 1997;99:2254-9.

31 Núňez NG, Andreani V, Crespo MI, et al. IFN $\beta$ Produced by TLR4-Activated Tumor Cells Is Involved in Improving the Antitumoral Immune Response. Cancer Research 2011;72:592-603.

32 Cursio $R$, Colosetti $\mathrm{P}$, Auberger $\mathrm{P}$, et al. Liver Apoptosis Following Normothermic IschemiaReperfusion: In Vivo Evaluation of Caspase Activity by FLIVO Assay in Rats. Transplantation Proceedings 2008;40:2038-41.

33 Fukata M, Shang L, Santaolalla R, et al. Constitutive activation of epithelial TLR4 augments inflammatory responses to mucosal injury and drives colitis-associated tumorigenesis. Inflammatory Bowel Diseases 2011;17:1464-73.

34 Kim T-H, Escudero S, Shivdasani RA. Intact function of Lgr5 receptor-expressing intestinal stem cells in the absence of Paneth cells. Proceedings of the National Academy of Sciences 2012;109:3932-7.

35 Rothenberg ME, Nusse Y, Kalisky T, et al. Identification of a cKit+ Colonic Crypt Base Secretory Cell That Supports Lgr5+ Stem Cells in Mice. Gastroenterology 2012.

36 Fukata M, Chen A, Klepper A, et al. Cox-2 Is Regulated by Toll-Like Receptor-4 (TLR4) Signaling: Role in Proliferation and Apoptosis in the Intestine. Gastroenterology 2006;131:862-77.

37 Brown SL, Riehl TE, Walker MR, et al. Myd88-dependent positioning of Ptgs2-expressing stromal cells maintains colonic epithelial proliferation during injury. The Journal of Clinical Investigation 2007;117:258-69.

38 Singh B, Berry JA, Shoher A, et al. COX-2 overexpression increases motility and invasion of breast cancer cells. International Journal of Oncology 2005;26:1393-9.

39 Müller-Decker K, Neufang G, Berger I, et al. Transgenic cyclooxygenase-2 overexpression sensitizes mouse skin for carcinogenesis. Proceedings of the National Academy of Sciences 2002;99:12483-8.

40 Nakamoto $\mathrm{N}$, Higuchi $\mathrm{H}$, Kanamori $\mathrm{H}$, et al. Cyclooxygenase-2 inhibitor and interferon- $\beta$ synergistically induce apoptosis in human hepatoma cells in vitro and in vivo. International Journal of Oncology 2006;29:625-35. 
41 Klampfer L, Huang J, Kaler P, et al. STAT1-independent inhibition of cyclooxygenase-2 expression by IFNY; a common pathway of IFNY-mediated gene repression but not gene activation. Oncogene 2007;26:2071-81. 